\title{
Statistical Power Law Tails in High-Energy Phenomena
}

\author{
Published online: 15 May 2009 - (c) Società Italiana di Fisica / Springer-Verlag 2009
}

The idea of non-extensive thermodynamics has been vividly debated for several years: promoters, appliers and opponents arguing with each other almost indefinitely. The Editors of The European Physical Journal A - Hadrons and Nuclei (EPJ A) realized at their last meeting that this topic is interesting enough to devote a topical issue to it, where the basic concepts and applications of these concepts in high-energy nuclear and particle physics are compiled in a rather pedagogical manner. Several important and characteristic promoters of the use of extended entropy formulas or other basic means in statistical physics for explaining power law tailed distributions have responded to our call to present a mini review of their own respective ideas. This process resulted in seven articles, which we proudly present in the following pages.

As a short guide for novel readers in these topics, we offer a comprised review of each contribution.

Constantino Tsallis is a most renown promoter of non-extensive thermodynamics and the application of nonconventional entropy formulas in statistical physics. A non-additive formula, often called the "Tsallis entropy" became a flag of this movement, which aims to understand and handle theoretically statistical systems with power law tailed distributions. Such distributions are abundant in complex natural or social systems; their interpretation is, however, manifold. The non-extensive approach offers a common framework to cope with all these phenomena. Having abundant followers and symphatizers on the one hand, but also some criticizers on the other hand, most basic concepts of statistical physics (from central limit theorem to the main laws of thermodynamics, from the role of noise to the generalization of the diffusion problem) have been recently reanalyzed in the light of his suggestion. Since nuclear and high-energy physics also meets phenomena where the statistical approach and thermodynamics is applied to systems under extreme conditions, and the "deeper" meaning of temperature-like parameters is often debated, it is proper to present a summary of these modern ideas for the readership of EPJ A. In the presented paper the author summarizes the basic idea of a particular non-additive entropy formula and its necessity to render the entropy extensive, in the sense of being proportional to the number of degrees of freedom, $N$, for special phase space filling patterns. Based on this the $q$-generalizations of familiar relations are tasked for the theoreticians to analyze; a particular example is the Central Limit Theorem, whose Levy-Gnedenko form is referred to in this paper. Alternative basic forms and their correspondences are clearly explained and the reader has a fast track to grasp the general complications in the non-extensive approach.

Christian Beck has pioneered the superstatistical approach to non-Boltzmannian energy spectra in different physical phenomena ranging from turbulence to cosmic rays. This approach interprets deviations from the canonical, exponential energy spectra as a distribution of the inverse slope, hence the name "superstatistics". Since the inverse slope is restricted to be positive, its limiting distribution is not a Gaussian, but an Euler-Gamma distribution. This leads to an effective, power law tailed energy distribution. In his review the author puts an emphasis on two important physical phenomena; namely on cosmic ray and electron-positron annihilation energy spectra. After introducing the general idea and arguing for the special form of superstatistical fluctuations, experimental data are reviewed together with theoretical curves. The value of the Tsallis parameter is derived in the high-energy limit.

Giorgio Kaniadakis has pioneered the surpassing of Boltzmann's Stosszahlansatz within the special relativity and proposes a new entropy, emerging as the relativistic generalization of the Boltzmann entropy. In statistical equilibrium, Kaniadakis entropy generates power law tailed distributions, which in the classical limit reduces to the MaxwellBoltzmann exponential distribution. In his paper he reviews the basic correspondence between two-particle correlation function and the thermodynamic entropy formula in the framework of kinetic theory. A clear explanation leads the reader from the generalization of the Stosszahlansatz with its factorizing form towards a more complicated form 
and to the generalization of the entropy formula established by Boltzmann, Gibbs and Shannon originally. The very formula suggested by the author is explained and established by a detailed analysis of composition formulas motivated by relativistic kinematics of momentum and energy addition. This way Kaniadakis arrives at an entropy formula which generates power law tailed momentum distributions in kinetic equilibrium. Mathematically, the generalization is carried out by the extensive use of the generalized (kappa-) logarithm and exponential functions. It is particularly important that the corresponding $H$-theorem is also presented for the kappa-entropy.

The authors Takeshi Kodama and Tomoi Koide are hydrodynamics experts in nuclear theory. In their paper they endeavour to catch a mechanism which can lead to a situation when non-extensive thermodynamics acts and TsallisPareto distributions form. The phenomenon is based on correlations which reduce the available phase space in a system. The example given by a constrained cluster formation is an easy-to-follow presentation of a model leading to the Tsallis-Pareto distribution, to the deformed logarithm and to Tsallis' entropy formula. Another example is given by coupled linear oscillators. This dynamical system is solved numerically as well as fitted by power law tailed distributions. The authors argue that systems showing Tsallis statistics may in general belong to a prethermalized state: isotropization of the momenta happens in a shorter timescale than the exponentiation of the energy. The authors close with interesting suggestions for further research on random interaction matrices.

Grzegorz Wilk and Zbigniew Wlodarczyk are experienced researchers in high-energy particle physics, one of their area of expertise being the application of information theory to the evaluation of experimental results on observed particle spectra. After realizing the fact that cut power law formulas fit different high-energy spectra amazingly well, a physical explanation is needed. The authors call our attention to temperature fluctuations, as a possible source of these phenomena. The paper is a very pedagogical review of some characteristic properties of high-energy particle spectra, which allows for an interpretation in the framework of non-extensive thermodynamics. To start with, it is shown that the non-extensivity parameter, $q$, can be connected to the width of temperature fluctuations in the source of these particle spectra. Then the non-exponential filling of the one-particle phase space is analyzed both for the longitudinal and transverse momenta separately. Besides the power law tail in transverse momentum distributions, the negative binomial multiplicity distribution is also explained by the imperfectness parameter, $q$, deviating from the uniform filling of the phase space. All these mathematical considerations are supported by a convincing amount of experimental data on $e^{+} e^{-}, p p, p \bar{p}$ and heavy-ion collisions at different bombarding energies.

Wanda Maria Alberico's and Andrea Lavagno's paper presents the non-extensive thermodynamics more from the application side in high-energy physics. Putting an emphasis on the quest for quark matter in relativistic heavy-ion collisions, both the concept of a quark gluon plasma and the relativistic non-extensive kinetic theory are introduced. A particular choice of representing local currents, which are fundamental for the hydrodynamics approach, is pedagogically presented and the relativistic equation of state for a formally ideal, but non-extensive plasma is discussed. As an application a model evolution equation for particle rapidity distributions is compared with experimental results on net proton (proton minus antiproton) spectra at RHIC and SPS heavy-ion collisions, and with predictions for the LHC. Studies of transverse momentum distribution and fluctuations round up the demonstration of the predictive power of non-extensive thermodynamics concepts. Finally, nuclear and quark matter equation of states are seeked for in this new framework. They shall become important ingredients in future studies of nuclear matter at high baryon densities.

The paper written by myself together with the young colleagues Gábor Purcsel and Károly Ürmössy reviews some new aspects of the origin and possible generalizations of non-extensive entropy formulas. Besides it presents the details of modelling and describing power law tailed transverse momentum spectra found in relativistic heavy-ion experiments. Starting with a simplified model of correlations the necessity of considering entropy and energy being non-extensive in the particle number is motivated. Quark matter at hadronization could exactly be such a system. A somewhat formal but straightforward section about abstract composition laws, deformed logarithms and corresponding general entropy formulas prepare the reader for the main argument: interaction energy under extreme relativistic kinematics may itself be a source of Tsallis composition rules and the corresponding cut power law distribution by applying the maximal entropy principle. Also a detailed description is given about the parton cascade model, which solves the Boltzmann equation under the energy conservation constraint with a non-additive two-particle energy composition rule. This leads to a power law tailed stationary distribution: this model generates numerically those spectra. The zeroth law of thermodynamics also has been studied in this framework in order to shed some light to former criticisms about the Tsallis entropy. It is demonstrated that some systems are able to equilibrate, even if they are described by a two-parameter entropy formula: besides the temperature, $T$, using another parameter, $q$. The last part deals with transverse momentum spectra of particles stemming from an assumed quark matter in heavy-ion collisions. The hadronization model is a simple quark coalescence, but experimental findings seem to cope with, so far.

Tamás S. Biró 